\title{
Sugammadex Reverses Safely and Effectively a Profound Neuromuscular Block in an Infant with Foreign Body Inhalation
}

\author{
Panagiotis Flamée", Jan Poelaert \\ Vrije Universiteit Brussel (VUB), Universitair Ziekenhuis Brussel (UZ Brussel), Department of \\ Anesthesia,Laarbeeklaan 101, 1090 Brussels, Belgium
}

*Corresponding Author: Panagiotis Flamée, Department of Anesthesia, Universitair Ziekenhuis Brussel, Brussels, Belgium, Email: Panagiotis.Flamee@uzbrussel.be

\begin{abstract}
Sugammadex (Bridion $\left.{ }^{\circledR}\right)$, a synthetic gamma-cyclodextrin molecule, is a selective-binding reversal drug that antagonizes neuromuscular blockade induced by steroidal neuromuscular blocking agents (NMBA) rocuronium- and vecuronium-bromide. According to current literature clinical studies with sugammadex in the pediatric population seems to be scarce. The absence of clinical studies contributes to restrained use.

A 13-month-old female infant presented at the emergency department with the diagnosis of aspiration of a foreign body. An urgent bronchoscopy under general anesthesia was planned for extraction, accordingly. Induction and maintenance of anesthesia was achieved totally intravenously with propofol and sufentanyl. Rocuronium was administered as a neuromuscular blocker in order to facilitate atraumatic introduction of the rigid bronchoscope and to provide adequate surgical conditions by preventing involuntary movements of the patient during extraction of the foreign body.

Removal of the foreign body was successful within minutes. Sugammadex was subsequently administered at a dose of $4 \mathrm{mg}_{\mathrm{kg}} \mathrm{kg}^{-1}$ in order to avoid unnecessary prolonged anesthesia and mechanical ventilation. The neuromuscular block was promptly reversed, regaining spontaneous ventilation. No adverse events occurred up until her discharge, the following day.
\end{abstract}

Conclusion: In this 13-month-old infant sugammadex promptly reversed the deep neuromuscular block induced by rocuronium. No adverse events were noted.

Keywords: Sugammadex, Rocuronium, Infant, Neuromuscular block reversal

\section{INTRODUCTION}

Sugammadex (Bridion®), a synthetic gammacyclodextrin molecule, is a selective binding reversal agent for neuromuscular blocks induced by steroidal neuromuscular blocking agents (NMBA) rocuronium- and vecuronium-bromide. It forms a complex, encapsulating the afore mentioned non-depolarizing NMBA, removing them from the neuromuscular junction and reversing hereby the muscle relaxant effect [1]. According to current literature reports of sugammadex in the pediatric population seems to be scarce [2]. Is it possible that offlabel administration of this NMBA-antidote in pediatric patients is underreported? The absence of clinical studies in this context could contribute to restrained use.

\section{MAterials AND MethodS}

A 13-month-old female presented at the emergency department with dyspnea and discrete wheezing. Hetero-anamnesis of the mother was suggestive for inhalation of a foreign body. She was alert but distressed, normally coloured with a normal capillary refill time. Lung auscultation revealed diminished vesicular breathing sound on the left side. Further clinical examination was unremarkable and there was no prior medical history. She had an American Society of Anesthesiologists classification I.

Flexible-fibroscopy confirmed the presence of a corpus alienum in the left main bronchus. An urgent rigid-bronchoscopy under general anesthesia was planned for extraction.

Induction of anesthesia was performed with propofol and sufentanyl. Rocuronium (0, $6 \mathrm{mg} \cdot \mathrm{kg}^{-1}$ ) was administered as a neuromuscular blocker in order to provide adequate surgical conditions and prevent undesired movements of 
the patient. Mechanical ventilation was secured with a high frequency jet-ventilator.

Removal of the foreign body was successful within a couple of minutes. In order to avoid unnecessary prolonged anesthesia, additional airway manipulation and further mechanical ventilation for undetermined time the anesthetists opted for sugammadex administration. Neuromuscular monitoring with acceleromyo graphy is possible in infants, but there are concerns about its accuracy on the measurements in infants [3]. Because of the very short procedural time and the known potent pharmacokinetic profile of rocuronium, which produces longer neuromuscular blocks in infants, as compared to children and adults, a $4 \mathrm{mg} \cdot \mathrm{kg}^{-1}$ dose of sugammadex was administered. Spontaneous ventilation was regained within two minutes of administration and the patient was awakened. The patient was monitored closely, in particular for recurarisation the first hours, since case reports have reported occurrence of this rare phenomenon. None of the known major or other adverse effects, noted in the package insert of sugammadex were reported during her stay at the postoperative care unit. Vital parameters were monitored overnight. The day after the patient was safely discharged.

\section{RESULTS AND DISCUSSION}

Inhalation of a foreign body can lead to a lifethreatening emergency, especially in children younger than three years old. Such events may confront anesthetists with challenging perioperative conditions.

Reversal of the effects of non-depolarizing NMBA is often achieved with acetylcholinesterase inhibitors (ACIs) that have undesired side effects. Moreover, an important condition is that the achieved block is not a profound one. The ACIs inability to reverse a profound block is a key shortcoming. Sugammadex is an innovative agent that overcomes this issue. It has the ability to form a complex with the steroidal non-depolarizing NMBA rocuronium and vecuronium, reducing the amount of NMBA available to bind to nicotinic cholinergic receptors in the neuro muscular junction, reversing in a dose- dependent way different levels of neuro muscular block [4]. On the other hand, its use in patients under 18 years old has not been established yet. Only one prospective study has analyzed short-term effects of sugammadex in pediatric population but the group of infants was very small, limiting hard conclusions [2]. There are several anecdotal reports, unraveling the potential clinical scenarios when sugammadex could have an advantageous value. The combination of rocuronium with sugammadex offered in this case a unique combination that can also be of great importance in emergency settings when a crushinduction is mandatory. Having the possibility to promptly reverse a deep block in the rare but potentially life-threatening scenario of cannot intubate-cannot ventilate (CICV), is an improvement in safety measures as also reported in the guidelines for a CICV scenario from the Delphi group and the second specialist group [5].

\section{Conclusions}

In this 13-month-old infant that underwent a bronchoscopic extraction of an inhaled foreign body sugammadex promptly reversed the deep block induced by rocuronium and no adverse events were noted up to her discharge. Prospective studies are needed to investigate the safety of sugammadex in infants.

\section{REFERENCES}

[1] Bom A, Hope F, Rutherford S, Thomson K. Preclinical pharmacology of sugammadex. J Crit Care. 2009; 24(1):29-35.

[2] Plaud B, Meretoja O, Hofmockel R, Raft J, Stoddart PA, van Kuijk JH, et al. Reversal of rocuronium-induced neuromuscular blockade with sugammadex in pediatric and adult surgical patients. Anesthesiology. 2009; 110(2):284-94.

[3] Driessen JJ, Robertson EN, Booij LHDJ. Acceleromyography in neonates and small infants: baseline calibration and recovery of the responses after neuromuscular blockade with rocuronium. European Journal of Anaesthesiology (EJA). 2005; 22(1):11-5.

[4] Naguib M, Brull SJ. Sugammadex: a novel selective relaxant binding agent. Expert Rev Clin Pharmacol. 2009; 2(1):37-53. 
Sugammadex Reverses Safely and Effectively a Profound Neuromuscular Block in an Infant with Foreign Body Inhalation

[5] Black AE, Flynn PER, Smith HL, Thomas ML, Wilkinson KA. Development of a guideline for the management of the unanticipated difficult airway in pediatric practice. Pediatric Anesthesia. 2015; 25(4):346-62.

Citation: Panagiotis Flamée, Jan Poelaert. Sugammadex Reverses Safely and Effectively a Profound Neuromuscular Block in an Infant with Foreign Body Inhalation. ARC Journal of Anesthesiology. 2017; 2(2):11-13. doi: dx.doi.org/10.20431/2455-9792.0202004.

Copyright: (C) 2017 Authors. This is an open-access article distributed under the terms of the Creative Commons Attribution License, which permits unrestricted use, distribution, and reproduction in any medium, provided the original author and source are credited. 